\title{
Nuclear Origins of Cell-to-Cell Variability
}

\author{
Z. WAKS AND P.A. SILVER \\ Department of Systems Biology, Harvard Medical School, Boston, Massachusetts 02115 \\ Correspondence: pamela_silver@hms.harvard.edu
}

\begin{abstract}
During the past decade, it has become increasingly evident that there is variation in the transcriptome of genetically identical cells, even when grown in homogenous environments. This cell-to-cell variability has been shown to have a central role in processes ranging from stem cell differentiation to chemotherapy resistance. Given that many genes display extensive heterogeneity in their messenger RNA (mRNA) abundance on a per cell basis, understanding the nuclear sources of this variability is important for our fundamental grasp of nuclear function and stands to have clinical manifestations. In this chapter, we assess the contribution of different transcription regimes, nuclear architecture dynamics, RNA polymerase elongation, and gene copy number to transcriptome heterogeneity. We also discuss techniques that can be used to quantify single-cell mRNA abundance and conclude by commenting on future research directions.
\end{abstract}

Just as identical twins are not completely identical, the same holds true at the single-cell level. It is well established that even genetically identical cells grown in uniform conditions have substantially varying transcription profiles (termed "transcriptome") (Bengtsson et al. 2005; Raj et al. 2006; Warren et al. 2006; Zenklusen et al. 2008). If heterogeneity in a population of cells is the norm, then what are its causes? As we highlight in this review, the extent of heterogeneity is a function of stochastic biochemical events and predetermined elements such as gene copy number.

A coordinated series of nuclear events is required in order to read the genetic code of DNA and convert it into an mRNA that will later be translated. As such, fluctuations in processes associated with this central dogma can have an impact on final mRNA abundance. Such fluctuations, in turn, lead to transcriptome heterogeneity among single cells. At the heart of transcriptome heterogeneity is the stochasticity associated with the process of transcription initiation. Stochasticity is derived from the randomness of intermolecular collisions that becomes relevant when small numbers of molecules determine the outcome of a reaction. Additionally, cell-to-cell variability can also arise from fluctuations in the activity of upstream pathway-specific factors. Although stochasticity in transcription and upstream fluctuations may explain much of the variability, understanding its extent requires knowledge of the specific nuclear events involved.

The phenotypes of eukaryotic cells are typically robust to fluctuations of their internal proteome (Kitano 2004, 2007). Nonetheless, these fluctuations can be more than simply insignificant noise. For example, a recent study demonstrated that the probability of a cancer cell being resistant to chemotherapy treatment is influenced by fluctuations of proteins regulating receptor-mediated apoptosis (Spencer et al. 2009). Furthermore, a series of studies demonstrated that fluctuations of the human immunodeficiency virus 1 (HIV-1) Tat protein are important in determining whether an infected cell will enter latency or lysogeny (Weinberger et al. 2005, 2008; Weinberger and Shenk 2007). From an engineering standpoint, expression heterogeneity can have impacts, from designing synthetic gene circuits (Drubin et al. 2007) to controlling stem cell differentiation (Chang et al. 2008).

This review focuses on the nuclear sources of gene-expression variability and, as such, on eukaryotic cells, although we note that many interesting studies have demonstrated substantial heterogeneity in bacteria as well (Balaban et al. 2004; Maamar et al. 2007; Mitchell et al. 2009). We assess the known nuclear sources of heterogeneity and discuss additional events that may also affect variability. Finally, we describe the techniques used to detect single-cell variation and comment on future research directions.

\section{TRANSCRIPTION COUPLED TO MRNA DEGRADATION IMPACTS HETEROGENEITY}

Eukaryotic transcription encompasses a complex series of events that begins with chromatin remodeling to increase promoter accessibility followed by the recruitment of many factors involved in transcription (Komili and Silver 2008; Hager et al. 2009). Because the molecular species involved in gene activation and transcription initiation are often present in low numbers, these events are inherently stochastic. Consequently, highly expressed and stable mRNAs are less prone to variability as the impact of stochasticity is reduced. Although oversimplified, models that characterize transcription often comprise two components (Fig. 1) (Peccoud and Ycart 1995; Kepler and Elston 2001; Paulsson 2005). First, gene activation is modeled as a random process where the promoter behaves in a binary fashion and can be in either an active $(\mathrm{ON})$ or inactive (OFF) state (Gardiner 2004). Second, once in the active configuration, transcription initiation is modeled as a random event; the chance of a single transcriptional event occurring at any given time is equal. Such behavior results in 


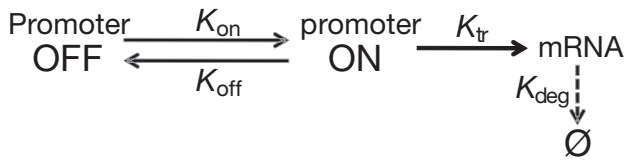

Figure 1. Model of mRNA transcription. $\left(K_{\text {on }}\right.$ and $\left.K_{\text {off }}\right)$ Gene activation and deactivation rate constants, respectively, $\left(K_{\text {tr }}\right)$ transcription initiation rate constant, $\left(K_{\mathrm{deg}}\right)$ mRNA degradation rate.

a Poisson distribution of transcription events in time and is modeled using the Gillespie algorithm (Gillespie 1977).

One important factor to consider when evaluating the gene activation-transcription model is the potential for time averaging of promoter behavior in the context of long-lived mRNAs. In mammalian cells, mRNAs are often stable for several hours (Yang et al. 2003; Sharova et al. 2009). Therefore, rapid switching between promoter states might have less impact on mRNA variability among cells because events associated with transcription and turnover would be averaged over time, and promoter activity can be approximated as percent ON. In contrast, a long period of promoter inactivity will result in very broad abundance distributions if an mRNA's lifetime is short relative to the time the promoter spends in its silent state (OFF state). This latter behavior is referred to as "bursting" or "pulsatile" because it is characterized by pulses of transcription separated by long durations of inactivity. Higher eukaryotes can tolerate transcriptional bursting owing to their long mRNA and protein half-lives. Although long half-lives reduce cell-to-cell variability, this comes at the cost of a slower response to upstream fluctuations. Overall, it is predicted that cell-to-cell variability is much larger in the presence of bursting transcription when compared with constitutive transcription $\left(K_{\text {on }}>>K_{\text {off }}\right)$ (Fig. 2).

Using the standard gene activation-transcription model, two distributions of single-cell mRNA abundance are expected. Constitutively active genes should follow a Poisson distribution. Likewise, mRNAs whose half-lives $\left(K_{\text {deg }}\right)$ are long relative to their promoter kinetics ( $K_{\text {on }}$ and $\left.K_{\text {off }}\right)$ should also follow Poisson distributions. In contrast, genes with long OFF states relative to the decay rate of their mRNA should exhibit wider distributions that are asymmetrically skewed toward higher abundances. As such, distributions of mRNA abundance for bursting genes often contain long tails that correspond to the small amount of actively transcribing cells per given time.

Bursts in transcription have been demonstrated in eukaryotes. Using single mRNA imaging, genes were shown to be transcribed in pulses in CHO cells and Drosophila (Raj et al. 2006; Pare et al. 2009). Transcriptional bursts have also been observed in Dictyostelium discoideum, mouse cells, and human cells (Levsky et al. 2002; Chubb et al. 2006; Voss et al. 2006). Consistent with the prevalence of transcriptional bursts, mRNA distributions in higher eukaryotes are typically very broad (Bengtsson et al. 2005; Raj et al. 2006; Warren et al. 2006; Pare et al. 2009) and follow a lognormal distribution (Bengtsson et al. 2005), i.e., their mRNA abundance histogram assumes a Gaussian distribution when plotted on a logarithmic scale. Lognormal distributions are common in nature and differ from normal distributions in that random independent effects are multiplicative rather than additive (Limpert et al. 2001).

In summary, the mode of transcriptional activation is an important factor in determining cell-to-cell variability of gene expression. In some cases bursts in transcription will be the rule, whereas in other cases mRNAs will be continuously produced. Some essential genes are enriched in areas of low nucleosome occupancy that are thought to correlate with more consistent transcription, suggesting that chromosomal gene positioning may have evolved, in part, to minimize variability of particular mRNAs by altering their transcription program (Batada and Hurst 2007).

\section{CHROMATIN REMODELING AND NUCLEAR LOCATION OF GENES AFFECT TRANSCRIPTION ON A CELL-BY-CELL BASIS}

Because nuclear architecture is not static, rearrangement within the nucleus has a major role in establishing transcription regimes. Just as isogenic cells vary in their
A

$$
\begin{aligned}
& \text { Constitutive transcription } \\
& \text { Mean transcription rate: } 1 \frac{\mathrm{mRNA}}{\mathrm{min}} \\
& \text { Bursting transcription } \\
& \text { Repeats of: } \underbrace{6 \frac{\mathrm{mRNA}}{\mathrm{min}}}_{10 \mathrm{~min}} \rightarrow \underbrace{0 \frac{\mathrm{mRNA}}{\mathrm{min}}}_{50 \mathrm{~min}}
\end{aligned}
$$
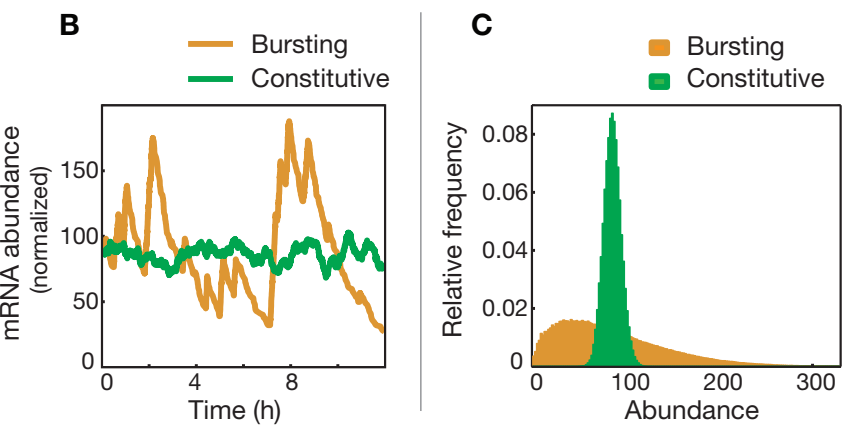

Figure 2. Bursting transcription is more variable than constitutive transcription. A computer simulation was used to estimate the mRNA abundance of an individual gene in $10^{7}$ cells. $(A)$ Model simulating both constitutive transcription ( $\left.1 \mathrm{mRNA} / \mathrm{min}\right)$ and bursting transcription. The bursting regime consisted of repetitive cycles of $6 \mathrm{mRNA} / \mathrm{min}$ for a mean duration of $10 \mathrm{~min}$ followed by $50 \mathrm{~min}$ of inactivity, on average. Both transcription paradigms yielded an average mRNA synthesis rate of $60 \mathrm{mRNA} / \mathrm{h}$ and a mean abundance of 87 mRNA molecules/cell. The mRNA half-life was $1 \mathrm{~h}$. $(B)$ Trajectories of mRNA concentration over time for a single cell in both transcription regimes. $(C)$ Histogram of mRNA abundance for $10^{7}$ cells. Bursting transcription results in more heterogeneity as quantified by the coefficient variation (CV, or standard deviation divided by the mean): 0.67 (bursting) compared with 0.11 (constitutive). 
transcriptional profile, heterogeneity in the nuclear architecture is also present in clonal populations. The chromatin state at the promoter of a gene has a pronounced effect on the likelihood of initiating transcription, and there are indications that the position of a locus within the nucleus may affect transcription.

In brief, chromatin remodeling is a phenomenon that consists primarily of histone acetylation and methylation and removal of these moieties (Gelato and Fischle 2008). Although the effects of methylation are complex and depend on the histone residue modified, acetylation is typically associated with gene activation because the steric volume of the alkyl group results in decondensation of the DNA (Gelato and Fischle 2008). This decondensation allows for binding of transcription factors and other proteins involved in transcription.

Large-scale chromatin state transitions can occur on the timescale of hours and therefore one would expect chromatin rearrangement to be a major source of promoter cycling between ON/OFF states (Tumbar et al. 1999). Indeed, studies have demonstrated that chromatin remodeling is largely responsible for bursting expression in yeast (Blake et al. 2003; Raser and O'Shea 2004). In support of this finding, it was later shown that two proximal genes exhibit simultaneous bursting, whereas two distal genes exhibit uncorrelated bursts (Becskei et al. 2005; Raj et al. 2006; Octavio et al. 2009). Furthermore, specific histone methylation events can dictate the pulse length and frequency of transcriptional bursts (Muramoto et al. 2010). Together, these observations suggest that chromatin state transitions have a role in transcriptional bursting.

A gene's intranuclear localization can correlate with its transcriptional activity, although the cause and effect of these phenomena are still unclear (Fig. 3) (Brown and Silver 2007; Papantonis and Cook 2010). The nuclear periphery is classically considered to be the domain of transcriptionally silenced loci (Sexton et al. 2007). Thus, cell-to-cell variability in the proximity of a gene to the nuclear envelope could lead to differences in transcription activity. However, there are exceptions because small subsets of highly transcribed genes are enriched at nuclear pores and several genes can be physically recruited to these complexes following active transcription (Brickner and Walter 2004; Casolari et al. 2004, 2005). It has been suggested that nuclear pore localization contributes to the hysteresis of certain inducible genes (Brickner 2009). In other cases, increasing evidence suggests that most transcription occurs in punctate nuclear positions known as transcription factories (Sutherland and Bickmore 2009). It has been shown that these subnuclear enrichments of active RNA polymerases can specialize in transcribing subsets of genes (Xu and Cook 2008; Schoenfelder et al. 2010). Despite their importance in gene expression, transcription factory abundance is substantially limited with respect to the number of expressed genes (Osborne et al. 2004). As such, proximity to an appropriate factory may influence a gene's transcription on a cell-by-cell basis. Long-range chromatin interactions can also modulate transcription because contacts between chromosomes are nonrandom and have been shown to regulate expression (Spilianakis et al. 2005; Zhao et al. 2006; Apostolou and
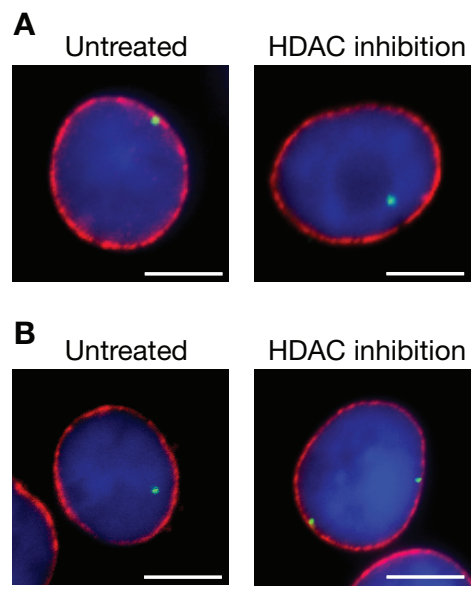

Figure 3. Genomic loci translocation following histone deacetylase (HDAC) inhibition. Fluorescent in situ hybridization (FISH) staining of genomic loci in HeLa cells was used to demonstrate locus translocation in response to HDAC inhibition (Brown et al. 2008). HDAC inhibition alters expression levels of large subsets of genes. (Green) FISH signal, (blue) 4',6-diamidino-2-phenylindole (DAPI) staining of the nucleus, (red) nuclear periphery. Bars, $5 \mu \mathrm{m}$. (A) Genomic locus that translocates away from the nuclear periphery following HDAC inhibition. $(B)$ Genomic locus that translocates to the nuclear periphery on HDAC inhibition. (FISH images adapted, with permission, from Brown et al. 2008.)

Thanos 2008). Taken together, cell-to-cell variability in the nuclear localization of a gene is likely to have a role in transcriptional heterogeneity.

\section{TRANSCRIPTIONAL ELONGATION AND VARIATION IN GENE EXPRESSION}

Human genes are often very long owing to the presence of introns, some of which are tens of thousands of base pairs long (Swinburne and Silver 2008). As a result, many human genes are longer than $100 \mathrm{~kb}$ and can take even hours to transcribe (Tennyson et al. 1995; Singh and Padgett 2009; Wada et al. 2009). When considering that polymerases not only vary in velocity but also pause during elongation, polymerase congestion behind slow or stalling elongation complexes becomes a possibility. These "traffic jams" could produce temporally separated pulses of mature mRNA (Swinburne et al. 2008; Saeki and Svejstrup 2009), thereby increasing cell-to-cell variability. However, the contribution of "traffic jam bursts" to increased heterogeneity is likely smaller than that of gene activation/inactivation because the interval between traffic jam bursts would typically be on a shorter timescale.

The elongation rate of a polymerase can, in principle, affect many aspects of transcription (de la Mata et al. 2003). Several studies have indicated that the polymerase elongation rate is in the range of $2-4 \mathrm{~kb} / \mathrm{min}$ in humans and $1-2 \mathrm{~kb} / \mathrm{min}$ in lower eukaryotes (Ardehali and Lis 2009). Elongation rates have been shown to be sequence dependent because mean velocities vary between genes, albeit to a small extent (Singh and Padgett 2009). Strikingly, single-molecule studies demonstrated that although the polymerization rate is approximately constant for an individual enzyme, there can be vast differences in elon- 
gation rate between individual polymerases on a particular gene (Davenport et al. 2000; Neuman et al. 2003; TolicNorrelykke et al. 2004). In fact, single-molecule in vitro studies of bacterial polymerases measured very broad velocity distributions with standard deviations of up to $42 \%$ of the mean (Davenport et al. 2000; Neuman et al. 2003; Tolic-Norrelykke et al. 2004). Whether this marked variability in elongation rate is present in a eukaryotic cell is still unknown; however, some variability is likely the case. Thus, a polymerase on the slower end of the spectrum may create a traffic jam, particularly in long genes.

RNA polymerase pausing occurs frequently during the course of elongation (Landick 2006). Although recent results have indicated that pausing is particularly widespread at promoter-proximal regions (Margaritis and Holstege 2008), stalling of the transcription elongation complex can also occur in a sequence-dependent manner throughout elongation (Gromak et al. 2006; Wada et al. 2009). Such inconsistency in elongation can lengthen a gene's transcription time. For example, a recent study in yeast observed that nonpausing polymerases required only $46 \mathrm{sec}$ to complete transcription of a $3.3-\mathrm{kb}$ reporter gene, compared with $517 \mathrm{sec}$ for their pausing counterparts (Darzacq et al. 2007). This represents a striking increase of $>10$ fold in transcription time. Consequently, whereas only $4 \%$ of the elongating polymerases paused, these enzymes were present on $26 \%$ of the actively transcribing genes (Darzacq et al. 2007).

A recent study in our laboratory has indicated that the traffic jam phenomenon may occur in eukaryotes (Swinburne et al. 2008). Using mouse fibroblasts, a synthetic negative-feedback loop was constructed using a yellow fluorescent protein (YFP) reporter gene that inhibits its own transcription when expressed. The synthetic gene contained introns of varying sizes, creating genes of 3,10 , and $19 \mathrm{~kb}$ in length. This circuit was found to generate oscillating pulses of protein expression. Mathematical analysis showed that pulsatile transcription caused by ON/OFF promoter-state transitions could only explain the length between pulses but not the increased standard deviation of burst length for the longer genes. Instead, this behavior was well explained by changes in the elongation rate of RNA polymerase.

Despite not being directly visualized in eukaryotes, a potential mechanism for traffic jams has recently been described. Following rear-end collision, the trailing polymerase undergoes substantial backtracking along the DNA template (Saeki and Svejstrup 2009). Afterward, forward elongation is reinitiated by transciption factor IIS (TFIIS)-mediated transcript cleavage of the excess mRNA (Saeki and Svejstrup 2009). Although the details of this process are still not fully characterized, this proposed mechanism presents a conceptual framework for comprehending polymerase congestion and subsequent cell-tocell variation in transcript number.

\section{GENE COPY NUMBER}

The copy number of a gene can also impact the extent of cell-to-cell variability in its expression. Each copy of a gene is subject to its own specific noise, also known as intrinsic noise (Elowitz et al. 2002; Swain et al. 2002). Using this logic, noise sources that affect both copies are termed extrinsic (Elowitz et al. 2002; Swain et al. 2002). Extrinsic noise is derived from pathway-specific fluctuations or global differences in transcription capacity.

In general, the more independently behaving copies of a gene present, the less heterogeneity there will be due to averaging of their activity. This is especially relevant in the case of bursting transcription. To better grasp this effect, one can imagine a gene that is present in two independently behaving copies and that each copy actively transcribes $25 \%$ of the time. When coupled together, transcription now occurs $44 \%$ of the time. This example illustrates that the presence of multiple independently behaving copies of a bursting gene transitions its transcription toward a more constitutive mode. As a result, cell-to-cell variability decreases and assumes a more Poisson-like distribution with increasing gene copy number. With respect to constitutive genes, Poisson statistics predict that cell-to-cell variability is expected to decrease with the inverse square root of the gene copy number, assuming the transcription rate of each copy is the same.

\section{QUANTIFYING TRANSCRIPT HETEROGENEITY}

Cellular heterogeneity can effectively be measured using any technique that gives single-cell resolution. Accordingly, microscopy, cell sorting, and reverse-transcriptionpolymerase chain reaction (RT-PCR) methods have been used to quantify heterogeneity at both the mRNA and protein level. Although these approaches each have their own advantages, they are not always sufficiently sensitive for the absolute quantification of single-cell mRNA transcript abundance. Here, we review several methods that can be used for this purpose.

Single-cell RT-PCR methods have been used to determine mRNA distributions for select genes (Fig. 4A) (Bengtsson et al. 2005; Warren et al. 2006). These approaches involve several enzymatic steps including lysis of an individual cell, reverse transcription, and PCR amplification. As such, they are subject to loss due to the multiple processing steps, and their accuracy may be further reduced by amplification bias. Given these limitations, PCR-based approaches are ideally suited for quantifying higher-abundance transcripts (Bengtsson et al. 2008), with the exception of digital PCR (Vogelstein and Kinzler 1999). In this method, the complementary DNA (cDNA) of an individual cell is diluted across a large number of separate PCRs such that each reaction has at most one template transcript for a given gene. The amount of transcripts per cell is determined by counting the number of positive PCRs as quantified by inclusion of a fluorescent reporter. Despite being technically challenging, digital PCR circumvents the amplification noise associated with the other PCR methods and has thus been used to measure mRNAs with mean abundances as low as 3.7 per cell (Warren et al. 2006). In the future, PCR methods 
A

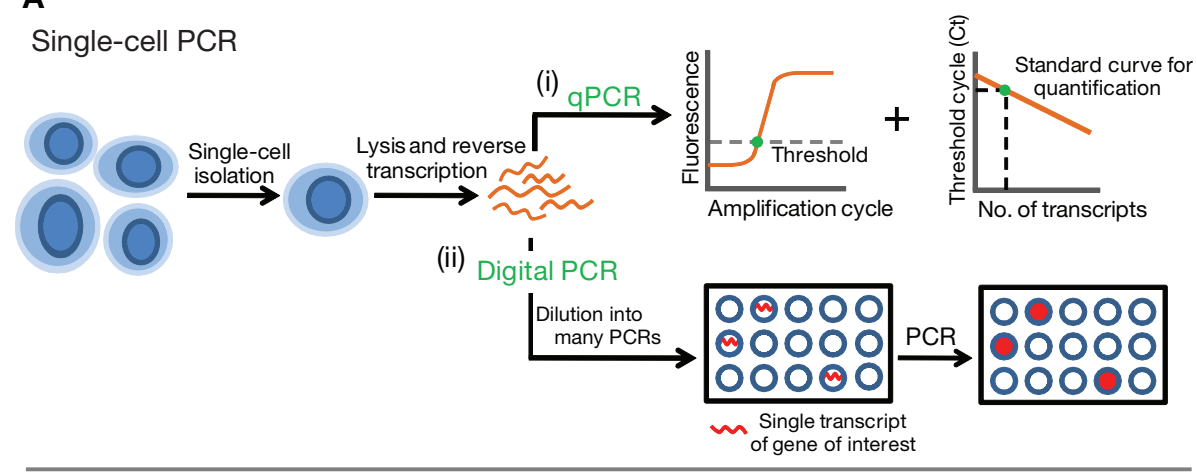

B

Single-molecule FISH

(i) 5' AAA ${ }^{\prime}$

(ii)
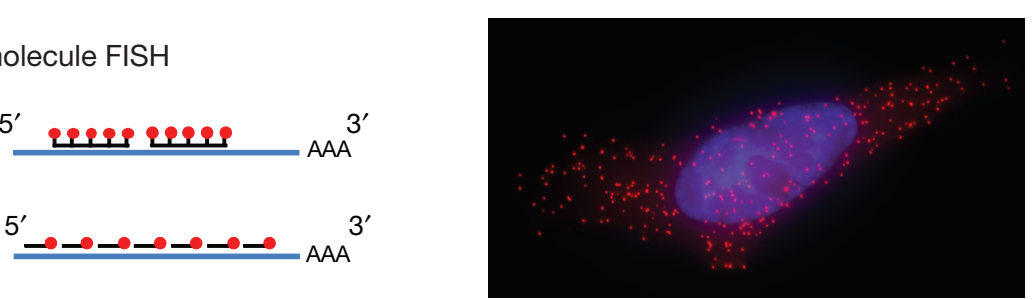

Figure 4. Methods for quantifying single-cell mRNA abundance. (A) PCR-based methods. Single cells are isolated, lysed, and reverse transcribed to produce cDNA. At this stage, either quantitative PCR (qPCR) or digital PCR is performed to assay transcript abundance. In the qPCR approach (i), traditional qPCR is coupled to a standard curve for quantification. The standard curve is attained by performing qPCR on the purified PCR product of the gene of interest using serial dilutions. In digital PCR (ii), the cDNA is diluted into hundreds or thousands of PCR reactions such that almost all contain either zero or one mRNA transcript. After thermal cycling, the amount of positive reactions represents the single-cell abundance of that mRNA. (B) Single-molecule FISH (smFISH) can be performed using (i) a handful of long, multiply labeled oligonucleotide probes (Femino et al. 1998) or (ii) dozens of short, singly labeled oligos (Raj et al. 2008). The long probes are typically $\sim 50 \mathrm{bp}$, in contrast to $\sim 20 \mathrm{bp}$ for their short counterparts. The image corresponds to smFISH for Caprin1 in HeLa cells using the Raj et al. method (P Silver et al., unpubl.). (Red speckles) Individual Caprin1 mRNA molecules. The image is a maximum intensity merge of 37 focal planes that span the entire volume of the cell. (Blue) DAPI staining of the nucleus.

may be used for quantifying genome-wide mRNA distributions because expression profiling in individual cells has been achieved on several occasions (Iscove et al. 2002; Kamme et al. 2003; Tietjen et al. 2003). Such global insights will be extremely valuable in discovering trends in heterogeneity, although they are currently limited by the high costs of performing many dozens of microarray or deep-sequencing assays as well as concerns regarding their accuracy.

Single-molecule imaging can be used to quantify transcript abundance by adapting FISH to a single-molecule format, a technique known as smFISH (Fig. 4B) (Femino et al. 1998; Raj et al. 2008). This method involves the hybridization of dozens of fluorescent molecules to select mRNAs such that each transcript yields an intense fluorescent signal that can be visualized. Using this approach, one can image multiple mRNAs simultaneously by using spectrally distinct fluorophores together with color mixing schemes. Because of its permeabilization, hybridization, and washing requirements, smFISH is a fixed cell technique. In this respect, although it is certainly possible to image individual mRNA transcripts in living cells using fluorescent RNA-binding proteins (Tyagi 2009), no live cell imaging technique can measure absolute mRNA abundance in eukaryotic cells owing to the rapid movement of mRNA molecules. As such, smFISH is currently the best imaging technique that offers this capability.

\section{CONCLUSIONS AND OUTLOOK}

In this review, we detail several nuclear sources that contribute to transcriptome variability among eukaryotic cells (Fig. 5). These include biochemical processes that are subject to stochastic noise and predetermined elements such as gene length and copy number. However, beyond the processes discussed here, gene expression encompasses additional nuclear events that are essential for efficient production of mature mRNA and could lead to further heterogeneity. For example, cell-to-cell differences in transcription fidelity may be important because errors in transcription can lead to premature degradation of a transcript through nuclear surveillance mechanisms. Likewise, mRNA export is a complex process involving dozens of proteins and thus there may be variability in export efficiency among cells (Mor et al. 2010). Finally, recent studies have shown that the transcriptome is far more diverse than previously thought (Mortazavi et al. 2008; Wilhelm et al. 2008), and our knowledge of its regulation remains a work in progress. For instance, the widespread prevalence of divergent transcription at promoter sites has only recently been appreciated (Core et al. 2008; Seila et al. 2008) and, as such, its regulatory role is largely uncharacterized. Given that numerous processes are involved in gene expression, more nuclear sources of cellular heterogeneity are likely to emerge. From a technological per- 


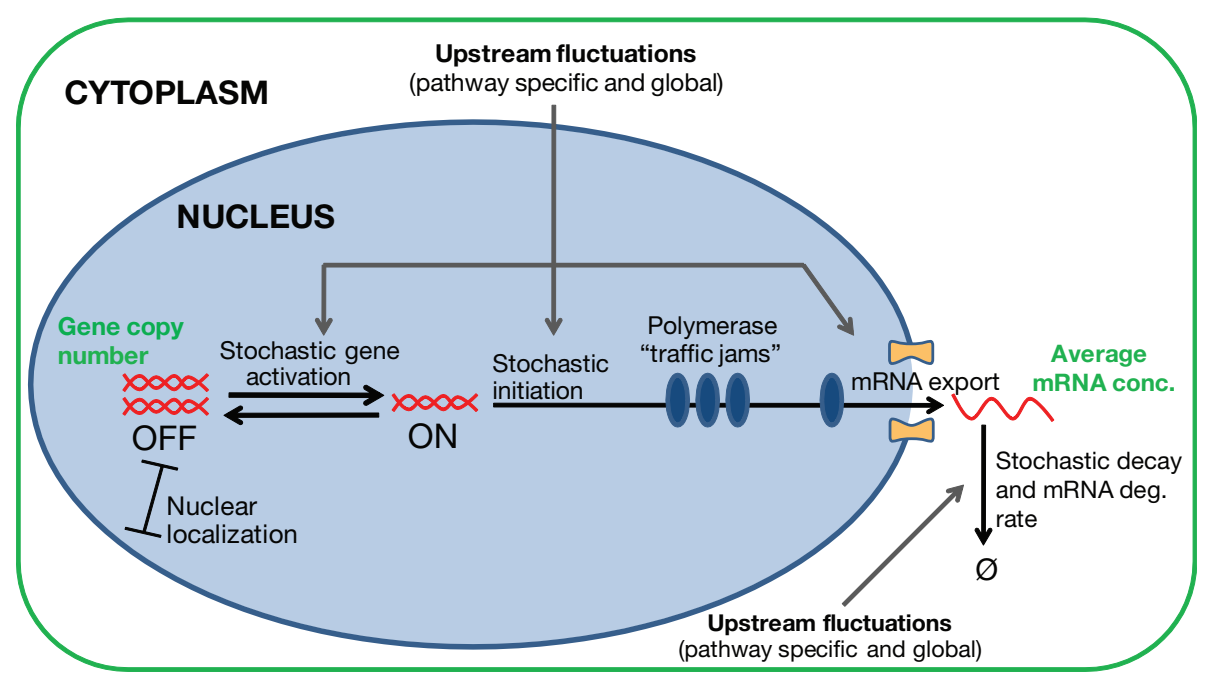

Figure 5. Schematic of nuclear sources of heterogeneity. The extent of cell-to-cell variability is determined by stochasticity in the course of mRNA synthesis and degradation (black) and by predetermined elements, such as gene copy number and average mRNA abundance (green). Average mRNA abundance is a function of transcription and degradation kinetics. mRNAs with long half-lives relative to their promoter activation/inactivation kinetics decrease heterogeneity by time averaging of promoter behavior. Upstream fluctuations can further increase cell-to-cell variability by impacting transcription and/or mRNA degradation.

spective, the field stands to benefit from the continuous improvement of single-molecule technologies, particularly those that enable live cell mRNA imaging. Such techniques will enable us to precisely characterize mRNA abundance and dynamics on a cell-by-cell basis.

The past decade has been host to a large number of studies that substantially enhanced our knowledge of eukaryotic cell-to-cell variability and its origins. Although cells have evolved to be robust in order to cope with the inevitable fluctuations in their transcriptome and proteome, heterogeneity is not always inconsequential. As mentioned previously, several studies have linked geneexpression heterogeneity with cancer treatment and HIV progression (Weinberger et al. 2005; Spencer et al. 2009), but the role of stochasticity and heterogeneity in disease manifestation and progression is still largely uncharacterized. For example, what underlying variations cause some cells to transform following carcinogen exposure and others to remain quiescent? It remains difficult to distinguish the extent to which environmental factors rather than cellular heterogeneity contribute to pathologies that seem to occur by chance, such as some cancers and autoimmune diseases. However, because stochastic gene expression has already been linked with cancer, HIV, aging, and T-cell activation (Weinberger et al. 2005; Bahar et al. 2006; Feinerman et al. 2008; Spencer et al. 2009), the role of expression heterogeneity in disease is clearly underappreciated. Given the clinical importance of cellular heterogeneity together with the presence of outstanding questions regarding its sources, we anticipate more fundamental discoveries to be made in the years to come.

\section{ACKNOWLEDGMENTS}

We are grateful to Buz Barstow, Andrew Giessel, Shay Tal, Dann Huh, Thomas Armel, and Joseph Shih for help- ful discussions and critical reading of the manuscript. Z.W. and P.A.S. acknowledge support from National Institutes of Health grants GM36373 and GM57476.

\section{REFERENCES}

Apostolou E, Thanos D. 2008. Virus infection induces NF-kB-dependent interchromosomal associations mediating monoallelic IFN- $\beta$ gene expression. Cell 134: 85-96.

Ardehali MB, Lis JT. 2009. Tracking rates of transcription and splicing in vivo. Nat Struct Mol Biol 16: 1123-1124.

Bahar R, Hartmann CH, Rodriguez KA, Denny AD, Busuttil RA, Dolle ME, Calder RB, Chisholm GB, Pollock BH, Klein CA, et al. 2006. Increased cell-to-cell variation in gene expression in ageing mouse heart. Nature 441: 1011-1014.

Balaban NQ, Merrin J, Chait R, Kowalik L, Leibler S. 2004. Bacterial persistence as a phenotypic switch. Science 305: 1622 1625 .

Batada NN, Hurst LD. 2007. Evolution of chromosome organization driven by selection for reduced gene expression noise. Nat Genet 39: 945-949.

Becskei A, Kaufmann BB, van Oudenaarden A. 2005. Contributions of low molecule number and chromosomal positioning to stochastic gene expression. Nat Genet 37: 937-944.

Bengtsson M, Stahlberg A, Rorsman P, Kubista M. 2005. Gene expression profiling in single cells from the pancreatic islets of Langerhans reveals lognormal distribution of mRNA levels. Genome Res 15: 1388-1392.

Bengtsson M, Hemberg M, Rorsman P, Stahlberg A. 2008. Quantification of mRNA in single cells and modelling of RT-qPCR induced noise. BMC Mol Biol 9: 63.

Blake WJ, Kaern M, Cantor CR, Collins JJ. 2003. Noise in eukaryotic gene expression. Nature 422: 633-637.

Brickner JH. 2009. Transcriptional memory at the nuclear periphery. Curr Opin Cell Biol 21: 127-133.

Brickner JH, Walter P. 2004. Gene recruitment of the activated INO1 locus to the nuclear membrane. PLoS Biol 2: e342.

Brown CR, Silver PA. 2007. Transcriptional regulation at the nuclear pore complex. Curr Opin Genet Dev 17: 100-106.

Brown CR, Kennedy CJ, Delmar VA, Forbes DJ, Silver PA. 2008. Global histone acetylation induces functional genomic reorganization at mammalian nuclear pore complexes. Genes Dev 22: 627-639. 
Casolari JM, Brown CR, Komili S, West J, Hieronymus H, Silver PA. 2004. Genome-wide localization of the nuclear transport machinery couples transcriptional status and nuclear organization. Cell 117: 427-439.

Casolari JM, Brown CR, Drubin DA, Rando OJ, Silver PA. 2005. Developmentally induced changes in transcriptional program alter spatial organization across chromosomes. Genes Dev 19: 1188-1198.

Chang HH, Hemberg M, Barahona M, Ingber DE, Huang S. 2008. Transcriptome-wide noise controls lineage choice in mammalian progenitor cells. Nature 453: 544-547.

Chubb JR, Trcek T, Shenoy SM, Singer RH. 2006. Transcriptional pulsing of a developmental gene. Curr Biol 16: 1018-1025.

Core LJ, Waterfall JJ, Lis JT. 2008. Nascent RNA sequencing reveals widespread pausing and divergent initiation at human promoters. Science 322: 1845-1848.

Darzacq X, Shav-Tal Y, de Turris V, Brody Y, Shenoy SM, Phair RD, Singer RH. 2007. In vivo dynamics of RNA polymerase II transcription. Nat Struct Mol Biol 14: 796-806.

Davenport RJ, Wuite GJ, Landick R, Bustamante C. 2000. Singlemolecule study of transcriptional pausing and arrest by E. coli RNA polymerase. Science 287: 2497-2500.

de la Mata M, Alonso CR, Kadener S, Fededa JP, Blaustein M, Pelisch F, Cramer P, Bentley D, Kornblihtt AR. 2003. A slow RNA polymerase II affects alternative splicing in vivo. Mol Cell 12: $525-532$.

Drubin DA, Way JC, Silver PA. 2007. Designing biological systems. Genes Dev 21: 242-254.

Elowitz MB, Levine AJ, Siggia ED, Swain PS. 2002. Stochastic gene expression in a single cell. Science 297: 1183-1186.

Feinerman O, Veiga J, Dorfman JR, Germain RN, Altan-Bonnet G. 2008. Variability and robustness in T cell activation from regulated heterogeneity in protein levels. Science 321: 1081-1084.

Femino AM, Fay FS, Fogarty K, Singer RH. 1998. Visualization of single RNA transcripts in situ. Science 280: 585-590.

Gardiner CW. 2004. Handbook of stochastic methods for physics, chemistry, and the natural sciences. Springer-Verlag, Berlin.

Gelato KA, Fischle W. 2008. Role of histone modifications in defining chromatin structure and function. Biol Chem 389: 353-363.

Gillespie DT. 1977. Exact stochastic simulation of coupled chemical-reactions. J Phys Chem 81: 2340-2361.

Gromak N, West S, Proudfoot NJ. 2006. Pause sites promote transcriptional termination of mammalian RNA polymerase II. Mol Cell Biol 26: 3986-3996.

Hager GL, McNally JG, Misteli T. 2009. Transcription dynamics. Mol Cell 35: 741-753.

Iscove NN, Barbara M, Gu M, Gibson M, Modi C, Winegarden N. 2002. Representation is faithfully preserved in global cDNA amplified exponentially from sub-picogram quantities of mRNA. Nat Biotechnol 20: 940-943.

Kamme F, Salunga R, Yu J, Tran DT, Zhu J, Luo L, Bittner A, Guo HQ, Miller N, Wan J, et al. 2003. Single-cell microarray analysis in hippocampus CA1: Demonstration and validation of cellular heterogeneity. J Neurosci 23: 3607-3615.

Kepler TB, Elston TC. 2001. Stochasticity in transcriptional regulation: Origins, consequences, and mathematical representations. Biophys J 81: 3116-3136.

Kitano H. 2004. Biological robustness. Nat Rev Genet 5: 826-837.

Kitano H. 2007. Towards a theory of biological robustness. Mol Syst Biol 3: 137.

Komili S, Silver PA. 2008. Coupling and coordination in gene expression processes: A systems biology view. Nat Rev Genet 9: $38-48$.

Landick R. 2006. The regulatory roles and mechanism of transcriptional pausing. Biochem Soc Trans 34: 1062-1066.

Levsky JM, Shenoy SM, Pezo RC, Singer RH. 2002. Single-cell gene expression profiling. Science 297: 836-840.

Limpert E, Stahel WA, Abbt M. 2001. Log-normal distributions across the sciences: Keys and clues. Bioscience 51: 341-352.

Maamar H, Raj A, Dubnau D. 2007. Noise in gene expression determines cell fate in Bacillus subtilis. Science 317: 526-529.

Margaritis T, Holstege FC. 2008. Poised RNA polymerase II gives pause for thought. Cell 133: 581-584.

Mitchell A, Romano GH, Groisman B, Yona A, Dekel E, Kupiec M, Dahan O, Pilpel Y. 2009. Adaptive prediction of environmental changes by microorganisms. Nature 460: 220-224.

Mor A, Suliman S, Ben-Yishay R, Yunger S, Brody Y, Shav-Tal Y. 2010. Dynamics of single mRNP nucleocytoplasmic transport and export through the nuclear pore in living cells. Nat Cell Biol 12: $543-552$.

Mortazavi A, Williams BA, McCue K, Schaeffer L, Wold B. 2008. Mapping and quantifying mammalian transcriptomes by RNASeq. Nat Methods 5: 621-628.

Muramoto T, Muller I, Thomas G, Melvin A, Chubb JR. 2010. Methylation of H3K4 Is required for inheritance of active transcriptional states. Curr Biol 20: 397-406.

Neuman KC, Abbondanzieri EA, Landick R, Gelles J, Block SM. 2003. Ubiquitous transcriptional pausing is independent of RNA polymerase backtracking. Cell 115: 437-447.

Octavio LM, Gedeon K, Maheshri N. 2009. Epigenetic and conventional regulation is distributed among activators of FLO11 allowing tuning of population-level heterogeneity in its expression. PLoS Genet 5: e1000673.

Osborne CS, Chakalova L, Brown KE, Carter D, Horton A, Debrand E, Goyenechea B, Mitchell JA, Lopes S, Reik W, et al. 2004 . Active genes dynamically colocalize to shared sites of ongoing transcription. Nat Genet 36: 1065-1071.

Papantonis A, Cook PR. 2010. Genome architecture and the role of transcription. Curr Opin Cell Biol 22: 371-376.

Pare A, Lemons D, Kosman D, Beaver W, Freund Y, McGinnis W. 2009. Visualization of individual Scr mRNAs during Drosophila embryogenesis yields evidence for transcriptional bursting. Curr Biol 19: 2037-2042.

Paulsson J. 2005. Models of stochastic gene expression. Phys Life Rev 2: $157-175$.

Peccoud J, Ycart B. 1995. Markovian modelling of gene product synthesis. Theor Popul Biol 48: 222-234.

Raj A, Peskin CS, Tranchina D, Vargas DY, Tyagi S. 2006. Stochastic mRNA synthesis in mammalian cells. PLoS Biol 4: e309.

Raj A, van den Bogaard P, Rifkin SA, van Oudenaarden A, Tyagi S. 2008. Imaging individual mRNA molecules using multiple singly labeled probes. Nat Methods 5: 877-879.

Raser JM, O'Shea EK. 2004. Control of stochasticity in eukaryotic gene expression. Science 304: 1811-1814.

Saeki H, Svejstrup JQ. 2009. Stability, flexibility, and dynamic interactions of colliding RNA polymerase II elongation complexes. Mol Cell 35: 191-205.

Schoenfelder S, Sexton T, Chakalova L, Cope NF, Horton A, Andrews S, Kurukuti S, Mitchell JA, Umlauf D, Dimitrova DS, et al. 2010. Preferential associations between co-regulated genes reveal a transcriptional interactome in erythroid cells. Nat Genet 42: 53-61.

Seila AC, Calabrese JM, Levine SS, Yeo GW, Rahl PB, Flynn RA, Young RA, Sharp PA. 2008. Divergent transcription from active promoters. Science 322: 1849-1851.

Sexton T, Schober H, Fraser P, Gasser SM. 2007. Gene regulation through nuclear organization. Nat Struct Mol Biol 14: 1049-1055.

Sharova LV, Sharov AA, Nedorezov T, Piao Y, Shaik N, Ko MS. 2009. Database for mRNA half-life of 19977 genes obtained by DNA microarray analysis of pluripotent and differentiating mouse embryonic stem cells. DNA Res 16: 45-58.

Singh J, Padgett RA. 2009. Rates of in situ transcription and splicing in large human genes. Nat Struct Mol Biol 16: 1128-1133.

Spencer SL, Gaudet S, Albeck JG, Burke JM, Sorger PK. 2009. Non-genetic origins of cell-to-cell variability in TRAIL-induced apoptosis. Nature 459: 428-432.

Spilianakis CG, Lalioti MD, Town T, Lee GR, Flavell RA. 2005. Interchromosomal associations between alternatively expressed loci. Nature 435: 637-645.

Sutherland H, Bickmore WA. 2009. Transcription factories: Gene expression in unions? Nat Rev Genet 10: 457-466.

Swain PS, Elowitz MB, Siggia ED. 2002. Intrinsic and extrinsic contributions to stochasticity in gene expression. Proc Natl Acad Sci 99: 12795-12800.

Swinburne IA, Silver PA. 2008. Intron delays and transcriptional 
timing during development. Dev Cell 14: 324-330.

Swinburne IA, Miguez DG, Landgraf D, Silver PA. 2008. Intron length increases oscillatory periods of gene expression in animal cells. Genes Dev 22: 2342-2346.

Tennyson CN, Klamut HJ, Worton RG. 1995. The human dystrophin gene requires 16 hours to be transcribed and is cotranscriptionally spliced. Nat Genet 9: 184-190.

Tietjen I, Rihel JM, Cao Y, Koentges G, Zakhary L, Dulac C. 2003. Single-cell transcriptional analysis of neuronal progenitors. Neuron 38: 161-175.

Tolic-Norrelykke SF, Engh AM, Landick R, Gelles J. 2004. Diversity in the rates of transcript elongation by single RNA polymerase molecules. J Biol Chem 279: 3292-3299.

Tumbar T, Sudlow G, Belmont AS. 1999. Large-scale chromatin unfolding and remodeling induced by VP16 acidic activation domain. J Cell Biol 145: 1341-1354.

Tyagi S. 2009. Imaging intracellular RNA distribution and dynamics in living cells. Nat Methods 6: 331-338.

Vogelstein B, Kinzler KW. 1999. Digital PCR. Proc Natl Acad Sci 96: 9236-9241.

Voss TC, John S, Hager GL. 2006. Single-cell analysis of glucocorticoid receptor action reveals that stochastic post-chromatin association mechanisms regulate ligand-specific transcription. Mol Endocrinol 20: 2641-2655.

Wada Y, Ohta Y, Xu M, Tsutsumi S, Minami T, Inoue K, Komura D, Kitakami J, Oshida N, Papantonis A, et al. 2009. A wave of nascent transcription on activated human genes. Proc Natl Acad Sci 106: 18357-18361.

Warren L, Bryder D, Weissman IL, Quake SR. 2006. Transcription factor profiling in individual hematopoietic progenitors by digital RT-PCR. Proc Natl Acad Sci 103: 17807-17812.

Weinberger LS, Shenk T. 2007. An HIV feedback resistor: Autoregulatory circuit deactivator and noise buffer. PLOS Biol 5: e9.

Weinberger LS, Burnett JC, Toettcher JE, Arkin AP, Schaffer DV. 2005. Stochastic gene expression in a lentiviral positive-feedback loop: HIV-1 Tat fluctuations drive phenotypic diversity. Cell 122: 169-182.

Weinberger LS, Dar RD, Simpson ML. 2008. Transient-mediated fate determination in a transcriptional circuit of HIV. Nat Genet 40: $466-470$.

Wilhelm BT, Marguerat S, Watt S, Schubert F, Wood V, Goodhead I, Penkett CJ, Rogers J, Bahler J. 2008. Dynamic repertoire of a eukaryotic transcriptome surveyed at single-nucleotide resolution. Nature 453: 1239-1243.

Xu M, Cook PR. 2008. Similar active genes cluster in specialized transcription factories. J Cell Biol 181: 615-623.

Yang E, van Nimwegen E, Zavolan M, Rajewsky N, Schroeder M, Magnasco M, Darnell JE, Jr. 2003. Decay rates of human mRNAs: Correlation with functional characteristics and sequence attributes. Genome Res 13: 1863-1872.

Zenklusen D, Larson DR, Singer RH. 2008. Single-RNA counting reveals alternative modes of gene expression in yeast. Nat Struct Mol Biol 15: 1263-1271.

Zhao Z, Tavoosidana G, Sjolinder M, Gondor A, Mariano P, Wang S, Kanduri C, Lezcano M, Sandhu KS, Singh U, et al. 2006. Circular chromosome conformation capture (4C) uncovers extensive networks of epigenetically regulated intra- and interchromosomal interactions. Nat Genet 38: 1341-1347. 


\section{$\$_{\mathrm{CSH}}^{\infty} \mathbf{\mathrm { CSH }}$ Cold Spring Harbor Symposia SYMPOSIA on Quantitative Biology}

\section{Nuclear Origins of Cell-to-Cell Variability}

Z. Waks and P.A. Silver

Cold Spring Harb Symp Quant Biol 2010 75: 87-94 originally published online March 29, 2011 Access the most recent version at doi:10.1101/sqb.2010.75.027

References This article cites 83 articles, 26 of which can be accessed free at: http://symposium.cshlp.org/content/75/87.full.html\#ref-list-1

License

Email Alerting Receive free email alerts when new articles cite this article - sign up in the box at the Service top right corner of the article or click here.

To subscribe to Cold Spring Harbor Symposia on Quantitative Biology go to: http://symposium.cshlp.org/subscriptions 